\title{
ON THE SELF-CONJUGATENESS OF DIFFERENTIAL FORMS ON BOUNDED DOMAINS
}

\author{
RICARDO ABREU BLAYA, JUAN BORY REYES, EFRÉN MORALES AMAYA, \\ AND JOSÉ MARÍA SIGARRETA ALMIRA
}

\begin{abstract}
Suppose $\Omega$ is a bounded domain in $\mathbb{R}^{n}$ with boundary $\Gamma$ and let $\mathcal{W}$ be a non-homogeneous differential form harmonic in $\Omega$ and Höldercontinuous in $\Omega \cup \Gamma$. In this paper we study and obtain some necessary and sufficient conditions for the self-conjugateness of $\mathcal{W}$ in terms of its boundary value $\left.\mathcal{W}\right|_{\Gamma}=\omega$.
\end{abstract}

\section{INTRODUCTION}

As is well known, a $k$-vector in $\mathbb{R}^{n}$ can be interpreted as a directed $k$-dimensional volume. Such entities were first considered by H. Grassmann in the second half of the 19th century. He thus created an algebraic structure which is now commonly known as the exterior algebra. At about the same time, Sir W. Hamilton invented his quaternion algebra which a. o. enabled him to represent rotations in three dimensional space. In his 1878 paper, W. K. Clifford united both systems into a single geometric algebra, later named after him.

Clifford analysis offers a function theory, which is a higher dimensional generalization of classical complex analysis in $\mathbb{R}^{2}$ (identifying $\mathbb{R}^{2}$ with $\mathbb{C}$ in the usual way) to Euclidean space $\mathbb{R}^{n}(n \geq 3)$. The theory is centred around the concept of monogenic functions, which constitute the kernel of a first order vector valued, rotation invariant, differential operator called the Dirac operator, which factorizes the Laplacian. The best general reference here is [5]; see also the brief review [9] of the content of this book.

On the other hand, the world-renowned theory of differential forms provides also a generalization in $\mathbb{R}^{n}$ of holomorphic functions of one complex variable. Although Clifford analysis seems to be truly appropriate to study differential forms by using Clifford algebras in a very beautiful way, this has been mentioned so far only in a

2010 Mathematics Subject Classification. 30G35.

Key words and phrases. Clifford analysis; Self-conjugate differential forms.

Two grants from Ministerio de Economía y Competitividad, Agencia Estatal de Investigación and Fondo Europeo de Desarrollo Regional, Spain, and CONACYT, México (MTM2016-78227C2-1-P, MTM2015-69323-REDT and FOMIX-CONACyT-UAGro 249818) supported in part this work. Juan Bory Reyes was partially supported by Instituto Politécnico Nacional in the framework of SIP programs (SIP20180225). 
few papers. For an overview of the main operator identities and properties of these objects in the Clifford analysis context we refer to [11] and the references quoted there.

In [6] the authors compare the language of differential forms and that of Clifford algebra valued multi-vector fields and shown that the spaces of smooth differential forms on the one hand, and smooth multi-vector functions (multi-vector fields) on the other are isomorphic in a natural way. Moreover the action of the operator $d-d^{*}$, where $d$ and $d^{*}$ are the differential and codifferential operator respectively, on the space of smooth $k$-forms is identified with the action (on the right) of the Dirac operator, which plays the role of the Cauchy-Riemann operator on the space of smooth $k$-vector fields. Meanwhile the action of the operator $d+d^{*}$ is identified with the action (on the left) of the Dirac operator. An extensive treatment of the Clifford algebras of differential forms, an elegant fashion of standard physics techniques, can be found in [13, 18.

In the present paper necessary and sufficient conditions are formulated such that a harmonic (in the real sense) differential form in a domain $\Omega$ is really a self-conjugate differential form there. Here again the full use of the isomorphism between the smooth differential forms on the one hand and smooth multi-vector functions on the other is the key point.

\section{Harmonic and Self-Conjugate differential forms. Statement of THE PROBLEM}

We will follow here the notations and conventions carried out in 6 .

Denoting by $\Lambda^{k} \mathbb{R}^{n}$ the space of alternating real-valued $k$-forms $(0 \leq k \leq n)$, the well known Grassmann algebra over $\mathbb{R}^{n}$ is the associative algebra

$$
\Lambda \mathbb{R}^{n}:=\bigoplus_{k=0}^{n} \Lambda^{k} \mathbb{R}^{n}
$$

endowed with the exterior multiplication $\wedge$.

A basis for $\Lambda^{k} \mathbb{R}^{n}$ is obtained as follows. Let $\left\{d x^{1}, d x^{2}, \ldots, d x^{n}\right\}$ be a basis for the dual space $\left(\mathbb{R}^{n}\right)^{*}$ of $\mathbb{R}^{n}$. If $A=\left\{i_{1}, \ldots, i_{k}\right\} \subset M=\{1, \ldots, n\}$ with $i_{1}<i_{2}<\cdots<i_{k}$, set

$$
d x^{A}:=d x^{i_{1}} \wedge d x^{i_{2}} \wedge \cdots \wedge d x^{i_{k}}
$$

and

$$
d x^{\emptyset}:=1
$$

Then for each $k=0,1, \ldots, n$, the set

$$
\left\{d x^{A}: A \subset M,|A|:=\operatorname{card}(A)=k\right\}
$$

is a basis for $\Lambda^{k} \mathbb{R}^{n}$.

Note that in particular

$$
\begin{gathered}
d x^{i} \wedge d x^{i}=0, \quad i=1, \ldots, n \\
d x^{i} \wedge d x^{j}+d x^{j} \wedge d x^{i}=0, \quad 1 \leq i \neq j \leq n .
\end{gathered}
$$


The following linear operators on $\Lambda^{k} \mathbb{R}^{n}$ play a fundamental role:

$$
d x^{j}: \Lambda^{k} \mathbb{R}^{n} \rightarrow \Lambda^{k+1} \mathbb{R}^{n}: d x^{j}\left[d x^{A}\right]:=d x^{j} \wedge\left(d x^{i_{1}} \wedge d x^{i_{2}} \wedge \cdots \wedge d x^{i_{k}}\right)
$$

and

where

$$
\widehat{d x^{j}}: \Lambda^{k} \mathbb{R}^{n} \rightarrow \Lambda^{k-1} \mathbb{R}^{n}: \widehat{d x^{j}}\left[d x^{A}\right]=\sum_{r=1}^{k}(-1)^{r} \delta_{j i_{r}} d x^{A \backslash\left\{i_{r}\right\}}
$$

$$
d x^{A \backslash\left\{i_{r}\right\}}:=d x^{i_{1}} \wedge \cdots \wedge d x^{i_{r-1}} \wedge d x^{i_{r+1}} \wedge \cdots \wedge d x^{i_{k}} .
$$

Here and in what follows we use the same symbol to denote the multiplication operator $d x^{j}$ and the differential form $d x^{j}$ itself. The way in which this operator acts on $d x^{A}$ justifies such abuse of notation. These operators are then extended to $\Lambda \mathbb{R}^{n}$ by linearity.

Direct computation shows that

$$
\begin{aligned}
d x^{j} \wedge d x^{r}+d x^{r} & \wedge d x^{j}=0, \quad \widehat{d x^{j}} \wedge \widehat{d x^{r}}+\widehat{d x^{r}} \wedge \widehat{d x^{j}}=0, \\
\widehat{d x^{j}} & \wedge d x^{r}+d x^{r} \wedge \widehat{d x^{j}}=-\delta_{r j} \mathcal{I}
\end{aligned}
$$

where $\mathcal{I}$ is the identity operator.

As usual (see [12]), a $k$-form in an open domain $\Omega$ of $\mathbb{R}^{n}$ is a map

$$
\omega_{k}: \Omega \mapsto \Lambda^{k} \mathbb{R}^{n}, \quad x \mapsto \sum_{|A|=k} \omega_{k, A}(x) d x^{A},
$$

where for each $A, \omega_{k, A}$ is a real-valued function in $\Omega$.

Such a map is said to belong to some class of functions on $\Omega$ if each of its components belongs to that class. In particular, we denote by $C^{1}\left(\Omega, \Lambda^{k} \mathbb{R}^{n}\right)$ the space of smooth $k$-forms in $\Omega$ and by $C^{0, \alpha}\left(\Gamma, \Lambda^{k} \mathbb{R}^{n}\right)\left(C\left(\Gamma, \Lambda^{k} \mathbb{R}^{n}\right)\right)$ the space of Hölder continuous (continuous) $k$-forms in $\Gamma$.

Furthermore, consider the fundamental linear operators on $C^{1}\left(\Omega, \Lambda^{k} \mathbb{R}^{n}\right)$, the exterior derivative and the co-derivative $d$ and $d^{*}$, respectively:

$$
d:=\sum_{j=1}^{n} d x^{j} \frac{\partial}{\partial x_{j}}, \quad d^{*}:=\sum_{j=1}^{n} \widehat{d x^{j}} \frac{\partial}{\partial x_{j}} .
$$

It is easy to see that $d^{2}=0, d^{* 2}=0$, and $d d^{*}+d^{*} d=-\Delta$, the Laplacian in $\mathbb{R}^{n}$.

The kernels of the exterior derivative $d$ and the co-derivative $d^{*}$ consist of the so-called closed $k$-forms and co-closed $k$-forms, respectively. A smooth $k$-form in $\Omega$, which is at the same time closed and co-closed, is called harmonic in $\Omega$ (in the sense of Hodge), i.e, a smooth $k$-form $\omega_{k}$ is said to be harmonic in $\Omega$ if and only if it satisfies in $\Omega$ the Hodge-de Rham system

$$
\left\{\begin{array}{l}
d \omega_{k}=0 \\
d^{*} \omega_{k}=0
\end{array}\right.
$$

Note that if $\omega_{k}$ is harmonic in an open domain $\Omega$, then automatically $\omega_{k}$ is also harmonic in the real sense, i.e., satisfies the Laplace equation $\Delta \omega_{k}=0$ in $\Omega$.

These definitions can be directly extended to non-homogeneous differential forms: $C^{1}\left(\Omega, \Lambda \mathbb{R}^{n}\right)$ will denote $\sum_{k=0}^{n} C^{1}\left(\Omega, \Lambda^{k} \mathbb{R}^{n}\right)$. 
If $\mathcal{W}=\sum_{k=0}^{n} \omega_{k} \in C^{1}\left(\Omega, \Lambda \mathbb{R}^{n}\right)$, where $\omega_{k} \in C^{1}\left(\Omega, \Lambda^{k} \mathbb{R}^{n}\right)$ is a $k$-form, we consider the action of the exterior derivative $d$ and the co-derivative $d^{*}$ as

$$
d \mathcal{W}=\sum_{0}^{n} d \omega_{k}
$$

and

$$
d^{*} \mathcal{W}=\sum_{0}^{n} d^{*} \omega_{k}
$$

respectively.

Following [10, a non-homogeneous differential form $\mathcal{W} \in C^{1}\left(\Omega, \Lambda \mathbb{R}^{n}\right)$ is said to be self-conjugate if

$$
d \mathcal{W}=d^{*} \mathcal{W}
$$

in $\Omega$, i.e., if

$$
d^{*} \mathcal{W}_{1}=0 ; \quad d \mathcal{W}_{k-1}=d^{*} \mathcal{W}_{k+1}(k=1, \ldots, n-1) ; \quad d \mathcal{W}_{n-1}=0 .
$$

Since $d d^{*}+d^{*} d=-\Delta$, we have that any self-conjugate differential form is also real harmonic.

Note that if $\mathcal{W}=\omega_{k}$, then it is self-conjugate if and only if it is harmonic in the sense of Hodge.

The main problem that we address in the remainder of the paper is: Given a real harmonic differential form $\mathcal{W}$ in $\Omega \subset \mathbb{R}^{n}$, being Hölder continuous in $\Omega \cup \Gamma$, under what condition on $\left.\mathcal{W}\right|_{\Gamma}=\omega$ is this differential form self-conjugate in $\Omega$ ?

The next two sections contain some basic notions and results coming from Clifford analysis that we shall need in order to reach the goal of the present article.

\section{Clifford Algebras and multi-Vectors}

The real Clifford algebra associated with $\mathbb{R}^{n}$ endowed with the Euclidean metric is the minimal enlargement of $\mathbb{R}^{n}$ to a real linear associative algebra $\mathbb{R}_{0, n}$ with identity, and such that $\underline{x}^{2}=-|\underline{x}|^{2}$, for any $\underline{x} \in \mathbb{R}^{n}$.

It thus follows that if $\left\{e_{j}\right\}_{j=1}^{n}$ is the standard basis of $\mathbb{R}^{n}$, then we must have that $e_{i} e_{j}+e_{j} e_{i}=-2 \delta_{i j}$. Every element $a \in \mathbb{R}_{0, n}$ is of the form $a=\sum_{A \subseteq N} a_{A} e_{A}$, $N=\{1, \ldots, n\}, a_{A} \in \mathbb{R}$, where $e_{\emptyset}:=e_{0}=1, e_{\{j\}}=e_{j}$, and $e_{A}=e_{\alpha_{1}} \cdots e_{\alpha_{k}}$ for $A=\left\{\alpha_{1}, \ldots, \alpha_{k}\right\}$, where $\alpha_{j} \in\{1, \ldots, n\}$ and $\alpha_{1}<\cdots<\alpha_{k}$, or still as $a=$ $\sum_{k=0}^{n}[a]_{k}$, where $[a]_{k}=\sum_{|A|=k} a_{A} e_{A}$ is a so-called $k$-vector $(k=0,1, \ldots, n)$.

If we denote the space of $k$-vectors by $\mathbb{R}_{0, n}^{k}$, then $\mathbb{R}_{0, n}=\sum_{k=0}^{n} \oplus \mathbb{R}_{0, n}^{k}$, leading to the identification of $\mathbb{R}^{n}$ with $\mathbb{R}_{0, n}^{1}$.

For a 1 -vector $\underline{x}$ and a $k$-vector $Y_{k}$, their product $\underline{x} Y_{k}$ splits into a $(k-1)$-vector and a $(k+1)$-vector, namely:

$$
\underline{x} Y_{k}=\left[\underline{x} Y_{k}\right]_{k-1}+\left[\underline{x} Y_{k}\right]_{k+1},
$$

where

$$
\left[\underline{x} Y_{k}\right]_{k-1}=\frac{1}{2}\left(\underline{x} Y_{k}-(-1)^{k} Y_{k} \underline{x}\right)
$$


and

$$
\left[\underline{x} Y_{k}\right]_{k+1}=\frac{1}{2}\left(\underline{x} Y_{k}+(-1)^{k} Y_{k} \underline{x}\right) .
$$

The inner and outer products between $\underline{x}$ and $Y_{k}$ are then defined by

$$
\underline{x} \bullet Y_{k}:=\left[\underline{x} Y_{k}\right]_{k-1} \quad \text { and } \quad \underline{x} \wedge Y_{k}:=\left[\underline{x} Y_{k}\right]_{k+1} .
$$

Notice also that

$$
\begin{aligned}
& {\left[\underline{x} Y_{k}\right]_{k-1}=(-1)^{k+1}\left[Y_{k} \underline{x}\right]_{k-1},} \\
& {\left[\underline{x} Y_{k}\right]_{k+1}=(-1)^{k}\left[Y_{k} \underline{x}\right]_{k+1} .}
\end{aligned}
$$

For further properties concerning inner and outer products between multi-vectors, we refer to [14].

Conjugation in $\mathbb{R}_{0, n}$ is defined by $\bar{a}:=\sum_{A} a_{A} \overline{e_{A}}$, where

$$
\overline{e_{A}}=(-1)^{k} e_{i_{k}} \cdots e_{i_{2}} e_{i_{1}}, \quad \text { if } e_{A}=e_{i_{1}} e_{i_{2}} \cdots e_{i_{k}} .
$$

In particular for a 1-vector $\underline{x}$ we have:

$$
\underline{\bar{x}}=-\underline{x} .
$$

The natural isomorphism

$$
\Theta: \mathbb{R}_{0, n}^{(k)} \mapsto \Lambda^{k} \mathbb{R}^{n}, \quad \sum_{|A|=k} Y_{k, A} e_{A} \mapsto \sum_{|A|=k} Y_{k, A} d x^{A}
$$

was introduced and applied for example in 6 , 3 .

The following identities can be easily verified (see [3]).

Lemma 3.1. Let $\underline{x}$ be a vector and $Y_{k}$ a k-vector. Then:

(1)

$$
\Theta\left(e_{j} \bullet Y_{k}\right)=\sum_{|A|=k} Y_{k, A} \widehat{d x^{j}}\left[d x^{A}\right],
$$

or, more generally,

$$
\Theta\left(\underline{x} \bullet Y_{k}\right)=\sum_{j=1}^{n} \sum_{|A|=k} x_{j} Y_{k, A} \widehat{d x^{j}}\left[d x^{A}\right] ;
$$

(2)

$$
\Theta\left(e_{j} \wedge Y_{k}\right)=\sum_{|A|=k} Y_{k, A} d x^{j} \wedge d x^{A},
$$

or, more generally,

$$
\Theta\left(\underline{x} \wedge Y_{k}\right)=\sum_{j=1}^{n} \sum_{|A|=k} x_{j} Y_{k, A} d x^{j} \wedge d x^{A} .
$$

Of course one can extend the action of the isomorphism $\Theta$ by linearity to the whole $\mathbb{R}_{0, n}$. 


\section{Clifford analysis and harmonic multi-Vector fields}

From now on, $\Omega$ stands for a Jordan domain, i.e. a bounded oriented connected open subset of $\mathbb{R}^{n}$, the boundary of which is a compact topological surface to be denoted by $\Gamma$. We shall assume $\Gamma$ to be smooth or piecewise smooth. This smoothness hypothesis on $\Gamma$ can be relaxed, but for simplicity we shall not use this possibility in any essential way.

Let $f$ be an $\mathbb{R}_{0, n}$-valued function in $\Omega$, say

$$
f(\underline{x})=\sum_{A} f_{A}(\underline{x}) e_{A}, \underline{x} \in \Omega,
$$

all $f_{A}$ thus being real valued.

We will denote by $C^{1}\left(\Omega, \mathbb{R}_{0, n}\right)$ the space of 1-time continuously differentiable

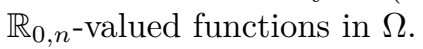

We say that $f$ is right (resp. left) monogenic in $\Omega$ if $F \mathcal{D}=0$ (resp. $\mathcal{D} F=0$ ) in $\Omega$, where $\mathcal{D}$ denotes the Dirac operator in $\mathbb{R}^{n}$ :

$$
\mathcal{D}=\sum_{j=1}^{n} e_{j} \partial_{x_{j}} .
$$

An important example of a function which is both right and left monogenic is the fundamental solution of the Dirac operator, given by

$$
E(\underline{x})=\frac{1}{A_{n}} \frac{\bar{x}}{|\underline{x}|^{n}}, \quad \underline{x} \in \mathbb{R}^{n} \backslash\{0\} .
$$

Hereby $A_{n}$ stands for the surface area of the unit sphere in $\mathbb{R}^{n}$.

The function $E(\underline{x})$ plays the same role in Clifford analysis as the Cauchy kernel does in complex analysis. For this reason it is also called the Cauchy kernel in $\mathbb{R}^{n}$.

Let $0<k \leq n-1$ be fixed. Then the space of $C^{1}$-functions from $\Omega$ into $\mathbb{R}_{0, n}^{k}$, called $k$-vector fields, is denoted by $C^{1}\left(\Omega, \mathbb{R}_{0, n}^{k}\right)$.

Notice that for $f_{k} \in C^{1}\left(\Omega, \mathbb{R}_{0, n}^{k}\right)$ a straightforward calculation leads to $\overline{\mathcal{D} f_{k}}=$ $\overline{f_{k}} \overline{\mathcal{D}}$ with $\overline{\mathcal{D}}=-\mathcal{D}$ and $\overline{f_{k}}=(-1)^{\frac{k(k+1)}{2}} f_{k}$. It thus follows that for an element in $C^{1}\left(\Omega, \mathbb{R}_{0, n}^{k}\right)$ the notions of left and right monogenicity coincide.

Consequently, we will call $f_{k} \in C^{1}\left(\Omega, \mathbb{R}_{0, n}^{k}\right)$ harmonic in $\Omega$ if either $\mathcal{D} f_{k}=0$ or $f_{k} \mathcal{D}=0$ in $\Omega$.

Moreover we notice that through the isomorphism $\Theta$, for $f_{k} \in C^{1}\left(\Omega, \mathbb{R}_{0, n}^{k}\right)$ and $\omega_{k}=\Theta f_{k} \in C^{1}\left(\Omega, \Lambda^{k} \mathbb{R}^{n}\right)$ we have $\mathcal{D} \wedge f_{k} \longleftrightarrow d \omega_{k}$ and $\mathcal{D} \bullet f_{k} \longleftrightarrow d^{*} \omega_{k}$ and are equivalent $(0<k<n-1)$

$$
\mathcal{D} f_{k}=0 \Longleftrightarrow \begin{cases}d \omega_{k} & =0 \\ d^{*} \omega_{k} & =0,\end{cases}
$$

i.e. $f_{k}$ harmonic in $\Omega$ is equivalent to saying that $\omega_{k}=\Theta f_{k}$ is a harmonic $k$-form in $\Omega$. 
More generally, let $\mathcal{W}=\sum_{0}^{n} \omega_{k}$ be a non-homogeneous differential form and consider the $\mathbb{R}_{0, n}$-valued function $F=\sum_{0}^{n} f_{k}$, where $\Theta f_{k}=\omega_{k}$; then $\mathcal{W}$ is a selfconjugate differential form in $\Omega$ if and only if $F$ is a right monogenic function in $\Omega$.

\section{CRiteria FOR MONOGEniCity}

Let us assume that $f$ belongs to the Hölder space $C^{0, \alpha}\left(\Gamma, \mathbb{R}_{0, n}\right), 0<\alpha<1$. The Cauchy transform $C_{\Gamma}$ and the Hilbert transform $H_{\Gamma}$ of $f$ are defined respectively by

$$
\begin{array}{ll}
C_{\Gamma} f(\underline{x})=\int_{\Gamma} E(\underline{y}-\underline{x}) \underline{\nu}(\underline{y}) f(\underline{y}) d \underline{y}, \quad \underline{x} \in \mathbb{R}^{n} \backslash \Gamma, \\
\left.H_{\Gamma} f(\underline{x})=\int_{\Gamma} E(\underline{y}-\underline{x}) \underline{\nu}(\underline{y}) f(\underline{y}) d \underline{y}\right), \quad \underline{x} \in \Gamma,
\end{array}
$$

where $\underline{\nu}(\underline{y})=\sum_{j=1}^{n} e_{j} \nu_{j}(\underline{y})$ is the unit normal vector on $\Gamma$ at the point $\underline{y}$.

It should be noticed that the last integral is taken in the principal value sense. Moreover, $C_{\Gamma} f$ is left monogenic in $\mathbb{R}^{m} \backslash \Gamma$. If $f$ is additionally left monogenic in $\Omega$, then by Cauchy's integral formula (see for instance [5], but it may be found in many other sources) we have

$$
f(\underline{x})=C_{\Gamma} f(\underline{x}), \quad \underline{x} \in \Omega .
$$

In [7] the authors give a condensed account of results connected to the Hilbert transform on the smooth boundary of a bounded domain in Euclidean spaces.

Let us now formulate important properties of $C_{\Gamma} f$ and $H_{\Gamma} f$.

(A) $H_{\Gamma} f \in C^{0, \alpha}\left(\Gamma, \mathbb{R}_{0, n}\right)$.

(B) (Sokhotski-Plemelj formula) For $\underline{z} \in \Gamma$,

$$
\lim _{\Omega \ni \underline{x \rightarrow} \underline{z}} C_{\Gamma} f(\underline{x})=\frac{1}{2}\left[H_{\Gamma} f(\underline{z})+f(\underline{z})\right] .
$$

A great number of original papers have been devoted to this subject. For the proof along classical lines we refer the reader to the pioneer work [15], whose author proved, in 1965, that the Cauchy transform has Hölder-continuous limit values for any Hölder-continuous densities and he obtained Plemelj-Sokhotski-type formulae.

We highlight the following important point. The claimed smoothness of the boundary $\Gamma$ for the validity of the items (A) and (B) has been known for many years but subsequent developments put the study of the above-mentioned items in the context of weaker restrictions on the boundary (see for instance [4, Theorem 1]). An optimal generalization of both assertions can be found in [1, Theorem 6].

We have assumed that $f \in C^{0, \alpha}\left(\Gamma, \mathbb{R}_{0, n}\right)$ and hence integrals are understood in the Riemann sense (proper or improper). If now $f \in L_{p}\left(\Gamma, \mathbb{R}_{0, n}\right)$ then one has to understand $C_{\Gamma} f$ as a Lebesgue integral, and the necessary changes can be easily made. For example, the (non-tangential) limits in (B) exist almost everywhere on $\Gamma$ with respect to the surface Lebesgue measure. An $L p$ formulation of (A) follows from standard Calderón-Zygmund theory and recalling that $C^{0, \alpha}\left(\Gamma, \mathbb{R}_{0, n}\right)$ is dense 
in $L_{p}\left(\Gamma, \mathbb{R}_{0, n}\right)$ by classical arguments. For a thorough treatment we refer the reader to $[16$.

The following theorem is basic in our next considerations. Its proof may be found in [2], but we include it here for the sake of completeness.

Theorem 5.1. Let $F: \Omega \cup \Gamma \rightarrow \mathbb{R}_{0, n}$ be a function such that $\left.F\right|_{\Gamma}=f$ belongs to $C^{0, \alpha}\left(\Gamma, \mathbb{R}_{0, n}\right)$. Then, the following are equivalent:

(i) $F$ is left monogenic in $\Omega$;

(ii) $F$ is harmonic in $\Omega$ and $H_{\Gamma} f=f$.

Proof. Suppose that $F$ is left monogenic in $\Omega$. From (5.1) we have $F(\underline{x})=C_{\Gamma} f(\underline{x})$ for $\underline{x} \in \Omega$. Now (B) yields

$$
f(\underline{x})=H_{\Gamma} f(\underline{x})+f(\underline{x}), \quad \underline{x} \in \Sigma .
$$

Consequently, $H_{\Gamma} f(\underline{x})=0$ for all $\underline{x} \in \Sigma$.

Conversely, assume that $F$ is harmonic in $\Omega$ and $H_{\Gamma} f=f$. Let us define

$$
G(\underline{x})= \begin{cases}C_{\Gamma} f(\underline{x}), & \underline{x} \in \Omega, \\ f(\underline{x}), & \underline{x} \in \Gamma .\end{cases}
$$

The function $G$ is left monogenic in $\Omega$ and hence harmonic in $\Omega$. By (A) and (B), $G$ is also continuous on $\Omega \cup \Gamma$. As $F-G$ is harmonic in $\Omega$ and $\left.(F-G)\right|_{\Gamma}=0$ it follows that $F(\underline{x})=C_{\Gamma} f(\underline{x})$ for $\underline{x} \in \Omega$.

Remark 5.2. As was mentioned before, we can extend the scope of the items (i) and (ii) to the much larger class of Lebesgue $p$-integrable functions (all formulas have to be reinterpreted), which makes it possible to carry our results with $L p$-data. In this sense, our approach generalizes and strengthens the standard result on the necessary and sufficient condition for the possibility to extend a given $L_{2}$-function from the surface $\Gamma$ to an $L_{2}$-monogenic function in the domain $\Omega$, see [7, Section 5], [12, Section 5] and [17, Chapter 3]. This goes back as far as [19, Theorem 95], where the very particular case of the half-plane is considered.

\section{Self-COnjugate Forms Revisited}

In this section we state and prove our main theorem, which gives a solution to the question asked at the end of Section 2. We retain the hypotheses on smoothness of $\Gamma$ for ease of comprehension.

Theorem 6.1. Let $\mathcal{W}: \Omega \cup \Gamma \rightarrow \Lambda \mathbb{R}^{n}$ be a non-homogeneous differential form such that $\left.\mathcal{W}\right|_{\Gamma}=\omega$ belongs to $C^{0, \alpha}\left(\Gamma, \Lambda \mathbb{R}^{n}\right)$. Then, the following are equivalent:

(i) $\mathcal{W}$ is a self-conjugate form in $\Omega$;

(ii) $\mathcal{W}$ is harmonic in $\Omega$ and

$$
\frac{2}{A_{n}} \int_{\Gamma} \sum_{j, r} \frac{z_{j}-\zeta_{j}}{|z-\zeta|^{n}} \nu_{r}(\zeta)\left(d z^{j} \wedge \widehat{d z^{r}}+\widehat{d z^{j}} \wedge d z^{r}\right) \omega(\zeta, d z) d \zeta=\omega(z, d z), \quad z \in \Gamma .
$$


Proof. Theorem 5.1 gives a direct and brief proof. Indeed, by Theorem 5.1 and keeping in mind the isomorphism $\Theta$ the proof is then reduced to verify the equality

$$
\Theta\left[H_{\Gamma} f\right]=\frac{2}{A_{n}} \int_{\Gamma} \sum_{j, r} \frac{z_{j}-\zeta_{j}}{|z-\zeta|^{n}} \nu_{r}(\zeta)\left(d z^{j} \wedge \widehat{d z^{r}}+\widehat{d z^{j}} \wedge d z^{r}\right) \omega(\zeta, d z) d \zeta
$$

where $\Theta[f]=\omega$, and this follows as a direct consequence of Lemma 3.1

Moreover, we obtain the following simple but important corollary.

Corollary 6.2. Let $\mathcal{W}_{k}: \Omega \cup \Gamma \rightarrow \Lambda^{k} \mathbb{R}^{n}$ be a $k$-differential form such that $\left.\mathcal{W}_{k}\right|_{\Gamma}=$ $\omega_{k}$ belongs to $C^{0, \alpha}\left(\Gamma, \Lambda^{k} \mathbb{R}^{n}\right)$. Then, the following are equivalent:

(i) $\mathcal{W}_{k}$ is harmonic in the sense of Hodge in $\Omega$;

(ii) $\mathcal{W}_{k}$ is real harmonic in $\Omega$ and

$$
\frac{2}{A_{n}} \int_{\Gamma} \sum_{j, r} \frac{z_{j}-\zeta_{j}}{|z-\zeta|^{n}} \nu_{r}(\zeta)\left(d z^{j} \wedge \widehat{d z^{r}}+\widehat{d z^{j}} \wedge d z^{r}\right) \omega_{k}(\zeta, d z) d \zeta=\omega_{k}(z, d z), \quad z \in \Gamma .
$$

\section{ACKNOWLEDGEMENT}

The authors are indebted to the anonymous referee for his/her valuable and constructive comments on the manuscript.

\section{REFERENCES}

[1] R. Abreu Blaya and J. Bory Reyes. Clifford analysis approach to a self-conjugate Cauchy type integral on Ahlfors regular surfaces. Ann. Polon. Math. 110 (2014), no. 2, 101-108. MR 3166251

[2] R. Abreu Blaya; J. Bory Reyes; D. Peña Peña and F. Sommen. Criteria for monogenicity of Clifford algebra-valued functions. Further progress in analysis, 167-174, World Sci. Publ., Hackensack, NJ, 2009. MR 2581620

[3] R. Abreu Blaya, J. Bory Reyes and M. Shapiro. The Cauchy transform for the Hodge/de Rham system and some of its properties. Georgian Math. J. 14 (2007), no. 1, 1-20. MR 2323369

[4] R. Abreu Blaya, J. Bory Reyes, O. Gerus and M. Shapiro. The Clifford-Cauchy transform with a continuous density: N. Davydov's theorem. Math. Methods Appl. Sci. 28 (2005), no. 7, 811-825. MR 2131752

[5] F. Brackx; R. Delanghe and F. Sommen. Clifford analysis. Research Notes in Mathematics, vol. 76. Pitman (Advanced Publishing Program), Boston, MA, 1982. MR 0697564.

[6] F. Brackx; R. Delanghe and F. Sommen. Differential forms and/or multi-vector functions. Cubo 7 (2005), no. 2, 139-169. MR 2186030.

[7] F. Brackx and H. De Schepper. The Hilbert transform on a smooth closed hypersurface. Cubo 10 (2008), no. 2, 83-106. MR 2431287.

[8] P. Caramuta and A. Cialdea. Some applications of the theory of self-conjugate differential forms. Bulletin TICMI 18 (2014), no. 2, 18-35. MR 3408567

[9] R. D. Carmichael. Book Review: Clifford analysis. Bull. Amer. Math. Soc. (N.S.) 11 (1984), no. 1, 227-240. MR 1567511

[10] A. Cialdea. On the theory of self-conjugate differential forms. Dedicated to Prof. C. Vinti (Perugia, 1996). Atti Sem. Mat. Fis. Univ. Modena 46 (1998), suppl., 595-620. MR 1645741.

[11] D. Eelbode and F. Sommen. Differential forms in Clifford analysis. Methods of complex and Clifford analysis, 41-69, SAS Int. Publ., Delhi, 2004. MR 2232586. 
[12] J. Gilbert and M. Murray. Clifford algebras and Dirac operators in harmonic analysis, Cambridge Studies in Advanced Mathematics, vol. 26. Cambridge University Press, Cambridge, 1991. MR 1130821

[13] D. Hestenes. Differential forms in geometric calculus. Clifford algebras and their applications in mathematical physics (Deinze, 1993), 269-285, Fund. Theories Phys., vol. 55. Kluwer Acad. Publ., Dordrecht, 1993. MR 1266877

[14] D. Hestenes and G. Sobczyk. Clifford algebra to geometric calculus, D. Reidel, Dordrecht, 1984. MR 0759340

[15] V. Iftimie. Fonctions hypercomplexes. Bull. Math. Soc. Sci. Math. R. S. Roumanie 9 (57) (1965), 279-332. MR 0217312.

[16] A. McIntosh. Clifford algebras, Fourier theory, singular integrals, and harmonic functions on Lipschitz domains. Clifford algebras in analysis and related topics (Fayetteville, 1993), 33-87. CRC, Boca Raton, 1996. MR 1383100

[17] M. Mitrea. Clifford wavelets, singular integrals, and Hardy spaces. Lecture Notes in Mathematics, vol. 1575. Springer-Verlag, Berlin, 1994. MR 1295843

[18] N. A. Salingaros and G. P. Wene. The Clifford algebra of differential forms. Acta Appl. Math. 4 (1985), no. 2-3, 271-292. MR 0802828

[19] E. C. Titchmarsh. Introduction to the theory of Fourier integrals. Third edition. Chelsea Publishing Co., New York, 1986. MR 0942661

R. Abreu

Grupo de Investigación de Análisis Complejo, Universidad de Holguín, Cuba.

rabreu@facinf.uho.edu.cu

J. Bory ${ }^{凶}$

SEPI-ESIME-ZAC, Instituto Politécnico Nacional, México.

juanboryreyes@yahoo.com

E. Morales

Universidad Autónoma de Guerrero, México.

efren.morales.amaya@cimat.mx

J. M. Sigarreta

Universidad Autónoma de Guerrero, México.

josemariasigarretaalmira@hotmail.com

Received: October 12, 2017

Accepted: November 13, 2018 